\title{
Rate and reasons for elective ventilation in patients undergoing intracranial tumour surgery
}

\author{
Charu Mahajan, Girija Prasad Rath', Manish Singh Sharma², Surya Kumar Dube', \\ Vanitha Rajagopalan ${ }^{1}$, Parmod Kumar Bithal ${ }^{1}$
}

\begin{abstract}
Background: Mechanical ventilation (MV) after neurosurgery is often decided by the preoperative neurological status of the patient. However, there is paucity of information regarding factors responsible for continuation of $M V$ in these patients. This study was carried out to identify the indications and risk factors for elective ventilation after intracranial tumour surgery. Materials and Methods: A prospective observational study was carried out on consecutive adult patients who underwent elective craniotomy for tumour excision, and postoperatively required MV. Data on anaesthesia technique, duration of anaesthesia and surgery, blood loss and transfusion and volume of fluids infused were noted. Intraoperative complications like tight brain, massive blood loss, brainstem handling, cranial nerve handling, haemodynamic instability, cardiac arrhythmias, venous air embolism, electrolyte abnormality and hypothermia were also recorded. Statistical analysis was done using Strata 9.0 software. Categorical data was analysed using Chi-square test or Fisher's exact test and continuous data by Student's t-test. Results: A total of 709 patients enrolled for the study over a period of one year out of which 347 patients (48.9\%) required continuation of MV during the postoperative period. The mean duration of MV was $29.7 \pm 39.7 \mathrm{hrs}$. The most common causes for postoperative MV were 'not responding to commands' (43.2\%), and neurosurgeon's advice (4I.8\%). The mean ICU and hospital stays were $92.2 \pm 134.0$ hrs and I3.8 \pm 16.5 days, respectively. $47.6 \%$ of patients who required postoperative MV on neurosurgeon's advice developed complications whereas it was $33.2 \%$ for those ventilated other reasons $(P<0.05)$. Glasgow outcome scale $(G O S)$ at discharge was poor in $12.4 \%$. On multivariate analysis, intraoperative blood transfusion, tracheostomy and duration of ventilation more than $48 \mathrm{hrs}$ were the independent risk factors associated with poor outcome. Conclusions: Although the neurosurgeon's advice for elective ventilation should not be ignored, but prolonged and avoidable MV may exacerbate the postoperative morbidities apart from increasing the cost of treatment. Hence, a complete understanding of intraoperative events, cerebral physiology and various factors influencing it during the perioperative period may not be overemphasised.
\end{abstract}

Key words: Complications, elective ventilation, neurosurgery, postoperative period

\begin{tabular}{|l|l|}
\hline \multicolumn{2}{|c|}{ Access this article online } \\
\hline Quick Response Code: & Website: \\
\hline & www.jnaccjournal.org \\
\cline { 2 - 2 } & \\
\hline & DOI: \\
\hline
\end{tabular}

\section{INTRODUCTION}

Mechanical ventilation (MV) is the life-support system provided until the patient deemed fit for tracheal extubation. Acute ventilatory failure, severe hypoxaemia and prophylactic ventilatory support are proposed as the indications for MV. ${ }^{[1]}$ Prophylactic (elective) ventilation provides adequate oxygenation, removes carbon dioxide, decreases work of breathing and buys time for the body to regain homeostasis before the

Department of Anaesthesiology, Sanjay Gandhi Post Graduate Institute of Medical Sciences, Lucknow, Uttar Pradesh, 'Department of Neuroanaesthesiology, All India Institute of Medical Sciences, New Delhi, India, ${ }^{2}$ Department of Neurosurgery, Mayo Clinic Health System, Minnesota, USA

Address for correspondence:

Dr. Girija Prasad Rath, Department of Neuroanaesthesiology, Neurosciences Centre, All India Institute of Medical Sciences, New Delhi - 110029 ,

India. E-mail: girijarath@yahoo.co.in 
patient can maintain spontaneous ventilation. The overriding benefit of postoperative elective ventilation is to prevent the early occurrence of respiratory and/or cardiovascular compromises leading to urgent tracheal reintubation. ${ }^{[2]}$ Gillespie et al. reported that $35 \%$ general surgical patients require postoperative ventilation because of haemodynamic instability, fluid overload, or residual effects of anaesthesia. ${ }^{[3]}$ The neurosurgical patients form a unique group who may require postoperative ventilation. The usual practice is to admit relatively fit patients who undergo an uneventful neurosurgical procedure to the intensive care unit (ICU) for vigilant observation. Patients with anticipated long operation time, extensive blood loss and high anaesthetic risk are the candidates for postoperative ICU admission. ${ }^{[4]}$

In our institution, numbers of patients are electively ventilated for different reasons after routine craniotomy. These patients in whom the tracheal extubation is delayed may have a longer ICU and hospital stay which adds to the higher cost of treatment. ${ }^{[5]}$ Paucity of information exists with regard to the defined reasons for the continuation of postoperative MV. This study was carried out to understand the risk factors and reasons of postoperative ventilation after elective intracranial tumour surgery.

\section{MATERIALS AND METHODS}

After obtaining approval from the Institute Ethics Committee, a prospective observational study was carried out comprising consecutive adult patients who underwent elective craniotomy for tumour excision and subsequently required ventilation in the postoperative period during a period of 1 year. Anaesthesia was induced with intravenous (IV) propofol $2-3 \mathrm{mg} / \mathrm{kg}$ and fentanyl $2 \mu \mathrm{g} / \mathrm{kg}$; tracheal intubation was facilitated by rocuronium $0.1 \mathrm{mg} / \mathrm{kg}$. Anaesthesia was maintained with isoflurane or sevoflurane in oxygen, $\mathrm{N} 2 \mathrm{O}$, and intermittent boluses of fentanyl and rocuronium. A patient was followed-up till discharge from the hospital, once it was decided by both the attending anaesthesiologist and neurosurgeon to continue MV during postoperative period. Patients who underwent emergency surgery or came to the operating room with a tracheal tube in situ were not included. Significant findings with regard to history, general physical and systemic examination, and investigations were recorded. Radiological information on tumour size, site and the presence of hydrocephalus, mass effect and midline shift were noted. Data regarding anaesthesia technique, patient position, duration of anaesthesia, blood loss, volume of fluids infused and blood transfusion were also noted. Intraoperative events like tight brain, massive blood loss, brainstem handling, cranial nerve handling, haemodynamic instability, cardiac arrhythmias, venous air embolism (VAE), electrolyte abnormalities and hypothermia were noted. Normothermia was maintained with the use of forced air-warming blanket. At the end of the surgery, the primary reason for not extubating the trachea was recorded, and the patients were shifted to ICU for elective ventilation. The mode of ventilation and sedation was at the discretion of the ICU physician. Postoperative complications such as pneumocephalus, haematoma, electrolyte imbalance, infarct, seizures, hydrocephalus, pneumonia, reintubation and tracheostomy were recorded. Duration of ventilatory support, length of ICU and hospital stay, and Glasgow outcome score (GOS) at discharge were also noted. The rate of postoperative ventilation was calculated at the end of the study period.

Statistical analysis was done using STATA 9.0 (College Station, TX, USA) software. Data is expressed as mean $\pm S D$ or number (percentage). Qualitative data was analysed using Chi-Square test or Fisher's exact test. Continuous data was analyzed by Student's t-test. Univariate and multivariate analysis was done by logistic regression to find the factors associated with poor outcome. The results were presented as Odds ratio $(95 \% \mathrm{CI})$. A $P$ value less than 0.05 was considered as significant.

\section{RESULTS}

A total of 709 patients underwent elective craniotomy for tumour surgery, out of which 347 patients (48.9\%) required postoperative ventilation [Table 1]. Preoperative hydrocephalus, mass effect and midline shift was present in $147(42.4 \%), 283(81.6 \%)$ and $108(31.1 \%)$ patients, respectively. In patients with infratentorial tumours, hydrocephalus was present in $99(57.6 \%)$ patients as compared to those with supratentorial tumours $48(27.1 \%)$ patients $(P<0.00)$. Tumours at supratentorial site caused midline shift in $66(37.8 \%)$ patients $(P<0.05)$ and mass effect in $147(84 \%)$ patients $(P>0.05)$. Tumour excision was

\section{Table 1: Demographic data of the patients}

\begin{tabular}{lc}
\hline Demographic variables & Mean \pm SD/ratio \\
\hline Age (years) & $33.3 \pm 17.2$ \\
Male:Female & $1.7: 1$ \\
Weight $(\mathrm{kg})^{*}$ & $54.4 \pm 17.6$ \\
Site & \\
Supratentorial (\%) & 50.2 \\
Infratentorial (\%) & 49.7 \\
Size $\left(\mathrm{cm}^{3}\right)^{*}$ & $84.4 \pm 114.6$ \\
\hline SD $=$ Stadard deviation
\end{tabular}

$\mathrm{SD}=$ Stadard deviation 
carried out in supine (161), sitting (34), lateral (98) and prone (54) positions. The mean intraoperative blood loss and blood transfused were $1098.6 \pm 1078.0 \mathrm{ml}$ and $12.0 \pm 17.9 \mathrm{ml} / \mathrm{kg}$, respectively. The mean duration of anaesthesia was $400 \pm 111.2 \mathrm{~min}$.

The mean duration of postoperative ventilation was $29.7 \pm 39.7 \mathrm{hrs}$; 'not responding to commands at the end of surgery' being the most common indication [Table 2]. All these patients received synchronised intermittent mandatory ventilation (SIMV) or pressure support ventilation (PSV) along with a combination of IV midazolam and fentanyl infusion for sedation. Various complications were reported during the postoperative period [Table 3], the commonest being pneumocephalus. Mean ICU and hospital stay was $92.2 \pm 134.0 \mathrm{hrs}$ and $13.8 \pm 16.5$ days, respectively. GOS at discharge was good (GOS 4-5) in $87.6 \%$ patients whereas it was poor in $12.4 \%$ patients [Table 4 ].

\begin{tabular}{lc} 
Table 2: Indications of postoperative ventilation \\
\hline Indication for elective ventilation & $\begin{array}{c}\text { No. of } \\
\text { patients (\%) }\end{array}$ \\
\hline Preoperative lower cranial nerve palsy & $36(10.4)$ \\
Haemodynamic instability & $7(2.0)$ \\
Brainstem handling & $41(11.8)$ \\
Massive blood loss & $27(7.8)$ \\
Intraoperative acute brain bulge & $14(4.0)$ \\
Residual tumour & $18(5.2)$ \\
Prolonged surgery (more than 8 hrs) & $44(12.7)$ \\
Not obeying commands & $15(43.2)$ \\
Neurosurgeon's advice & $145(41.8)$ \\
\hline
\end{tabular}

Table 3: Postoperative complications and secondary procedures*

\begin{tabular}{lc}
\hline Complications & No. of patients (\%) \\
\hline Hydrocephalus & $36(10.4)$ \\
Re-exploration* & $17(4.9)$ \\
Infarct & $22(6.3)$ \\
Meningitis & $13(3.8)$ \\
Haematoma & $42(12.1)$ \\
Pneumocephalus & $61(17.6)$ \\
Tension pneumocephalus & $3(0.9 \%)$ \\
Re-intubation* & $24(7.0)$ \\
Seizures & $37(10.7)$ \\
Pneumonia & $28(8.0)$ \\
Electrolyte abnormalities & $39(11.2)$ \\
Cardiac arrest & $8(2.3)$ \\
Tracheostomy* & $29(8.4)$
\end{tabular}

*Secondary procedures
Sixty-nine out of 145 patients $(47.6 \%)$ who required postoperative ventilation on neurosurgeon's advice developed complications. Among the rest other patients who required elective ventilation $33.2 \%$ developed complication $(P<0.05)$. However, the overall outcome at discharge was comparable between the two groups.

On univariate analysis, perioperative variables such as hydrocephalus, haematoma, infarct, reintubation, tracheostomy, meningitis, blood transfusion, ventilation for more than $48 \mathrm{hrs}$, tracheostomy and prolonged ICU stay were associated with poor outcome [Table 5]. Multivariate analysis confirmed intraoperative blood transfusion, duration of ventilation more than $48 \mathrm{hrs}$ and tracheostomy to be the independent risk factors for the poor outcome [Table 6].

\section{DISCUSSION}

Neurosurgical patients are different in that apart from cardiopulmonary complications which determine the requirement for postoperative ventilation; their neurological condition also affects this decision. Arterial hypertension or hypotension, increased venous pressure, hypercapnia and hypoxia may all exacerbate neuronal injury following neurosurgery. Smooth anaesthetic emergence and extubation, and the avoidance of emergency reintubation should therefore. always be aimed for. Systemic hypertension often accompanies

Table 4: Glasgow outcome scale score at discharge from hospital

\begin{tabular}{lc}
\hline Glasgow outcome scale score & No. of patients (\%) \\
\hline Death & $17(4.9)$ \\
Vegetative state & $8(2.3)$ \\
Severe disability & $18(5.2)$ \\
Moderate disability & $38(11.0)$ \\
Good recovery & $266(76.7)$ \\
\hline
\end{tabular}

Table 5: Postoperative variables associated with poor outcome (univariate analysis)

\begin{tabular}{lcc}
\hline Variables & $\begin{array}{c}\text { No. of } \\
\text { patients (\%) }\end{array}$ & P value \\
\hline Hydrocephalus & $9(21)$ & 0.01 \\
Haematoma & $12(28)$ & 0.001 \\
Infarct & $10(23.3)$ & 0.00 \\
Reintubation & $12(28)$ & 0.00 \\
Tracheostomy & $18(41.9)$ & 0.00 \\
Meningitis & $4(9.3)$ & 0.04 \\
Cardiac arrest & $1(2.3)$ & 0.008 \\
ICU stay more than $48 \mathrm{hrs}$ & $35(81.4)$ & 0.007 \\
\hline
\end{tabular}

Poor outcome: GOS 1-3, ICU = Intensive care unit 
Table 6: Multivariate analysis showing independent risk factors for postoperative ventilation

\begin{tabular}{lcc}
\hline Poor outcome & $\begin{array}{c}\text { Odds ratio } \\
(\mathbf{9 5 \%} \mathbf{C I})\end{array}$ & $\boldsymbol{P}$ value \\
\hline Intraoperative blood transfusion & $3.7(1.3-10.9)$ & 0.01 \\
Tracheostomy & $40.14(6.4-251.5)$ & 0.00 \\
Duration of ventilation more & $5.06(1.1-22.4)$ & 0.03 \\
than 48 hrs & & \\
\hline
\end{tabular}

Poor outcome: GOS 1-3, Cl = Confidence Interval

rapid emergence and may predispose to formation of intracranial haematoma. ${ }^{[6]}$ Delayed awakening at times may be encouraged, to limit the postoperative hyperdynamic and metabolic stress resulting from rapid reversal of anaesthetic state. It may also help in reducing the risk of hypoxia and hypercarbia, and allows achieving adequate surgical haemostasis. However, Bruder et al. observed early recovery and extubation after intracranial surgery to be associated with fewer metabolic and cardiovascular changes than a $2 \mathrm{hrs}$ delayed recovery..$^{[7]}$ This may be the result of higher residual opioid or propofol plasma level immediately after surgery.

The rate of elective ventilation was found to be very high $(48.9 \%)$ in our study. Several factors may have contributed to this. It is often presumed that with large-sized brain tumours, the perioperative course becomes turbulent. Surgery for removal of more than 30-mm brain tumour was associated with a higher incidence of delayed emergence in the early postoperative period ${ }^{[8]}$ Irrespective of size, tumours causing ischaemia or compression in the region of reticular activating system may cause delayed awakening. In our study, there was no relation between tumour size and the outcome probably due to the interplay of various other factors like site, relation to vital structures, intra-operative course and tumour histopathology. Moreover, radiological findings like presence of preoperative hydrocephalus, midline shift and mass effect were also not related to duration of elective ventilation or outcome.

At our centre, brain stem auditory evoked potentials are not monitored routinely during the posterior fossa surgery. Haemodynamic responses to surgical stimulation of brainstem structures are observed, clinically. Intra-operative brainstem handling may be manifested as sudden episodes of haemodynamic changes not explained by any other reason. More than two such unexplainable episodes during surgical resection in vicinity of brainstem, is considered as significant. Surgical manipulation close to lower cranial nerve (LCN) nuclei and vital centers in the brainstem may lead to retraction oedema and transient dysfunction. There may be loss of protective reflexes of the airway owing to LCN injury. ${ }^{[9]}$ Such patients were electively ventilated postoperatively so that the blunted response of cardio-respiratory centres and protective reflexes recover, over a period of time. The integrity of airway depends on intact function of cranial nerves such as VII, IX, X and XII. Swallowing dysfunction is related to abnormal IX, X, XII cranial nerve function. Thus, preoperative LCN dysfunction was another reason of delayed extubation. There is fear of aspiration of gastric contents in view of inability to protect one's airway. Delayed extubation, later in the ICU, can be done under more controlled conditions, thus avoiding urgent reintubation. The patients who are unfit for extubation, an early tracheostomy (7-10 days) is carried out as a routine practice.

Massive blood loss is associated with transfusion of large amount of IV fluids and blood products which leads to interstitial fluid shifts and generalised oedema. The patients were electively ventilated so that body regains homeostasis. Dilutional effects may also lead to coagulational disorders and defective haemostasis resulting in high probability of haematoma formation. In this study, it was found that patients with intraoperative blood loss more than 1 litre had significant chance of development of postoperative haematoma $(P<0.01)$.

Tight brain at dural closure indicates high probability of developing malignant brain oedema. Occurrence of hypercapnia following tracheal extubation may lead to increase in ICP in patients already having a tight brain. Although 34 patients presented with intraoperative tight brain, but a switch over to propofol infusion from inhalational anaesthetics helped in achieving adequate brain relaxation. An acute brain bulge was seen in 14 patients. All of these patients were electively ventilated during postoperative period. In refractory cases, decompressive craniectomy (DC) was carried out; three patients underwent DC in the same sitting. The patients require therapeutic maneuvers to achieve brain relaxation which can easily be achieved in the setting of elective ventilation. The decision to extubate the trachea relies on an unremarkable postoperative CT scan. Moreover, elective ventilation eliminates the possibility of an urgent reintubation which runs the risk of inducing secondary intracranial hypertension.

A large infiltrating tumour attached to vital structures or close to eloquent area may be incompletely resected. Postoperative oedema is especially severe when residual tumour is intentionally left. This may be explained by changes in regional blood flow due to autoregulatory failure in the tumour tissue. ${ }^{[10]}$ It is safe to electively ventilate such patients until the oedema resolves. The arteries within the residual tumour lack automatic spasticity when damaged and may continue to bleed. ${ }^{[11]}$ 
Thus, chance of haematoma formation is greater in the region of a residual tumour. Postoperative ventilation may help maintain low normal blood pressure.

VAE occurred in five patients with significant haemodynamic changes. These patients were efficiently managed and by the time surgery ended, no patient had unstable cardio-respiratory function. These patients were, however, decided to be electively ventilated.

Among all the indications for elective ventilation, neurosurgeon's advice may seem to be nonspecific. But interestingly, it constituted $41.8 \%$, making it the single most common primary indication in our study. Extensive surgery especially close to the vital structures probably makes the neurosurgeon hesitant for an early tracheal extubation. The surgeon may not achieve a satisfactory haemostasis in presence of continuous ooze, and hence, desire to have a CT scan prior to tracheal extubation. Inadvertent stimulation or injury to hypothalamus, cranial nerves or major vessels may also be the reason for surgeon's inclination for continuation of ventilation. Handling of major vessels may result in spasm or inadvertent injury manifested by infarct. Also, with pressure of time in operation theatre, the attending anaesthesiologist and surgeon may jointly decide to extubate the patient in ICU.

Out of the 145 patients who were electively ventilated on neurosurgeon's advice, $87(58.8 \%)$ patients had an apparently uneventful intraoperative course. It might be due to overcautious attitude of the operating neurosurgeons. The postoperative complication rate was more $(55.9 \%$ vs $44.1 \%)$ in patients who were ventilated on neurosurgeon's advice as compared to those who were ventilated for other reasons $(P=0.03)$. However, the outcome was comparable in between these two groups. It may be argued that most of these cases elective ventilation could have been avoided. However, the counter argument is that, the duration of ICU stay, hospital stay and GOS were comparable between the two groups; thus, indicating that neurosurgeon's advice for elective ventilation probably helped the patient.

Not obeying to verbal commands despite reversed from residual neuromuscular blockade may be due either to residual effect of anaesthesia or to neurosurgical reasons. However, none of the patients had any sign of opioid overdose, discernible end-tidal volatile agent, or residual muscle relaxant effect was observed. As per our practice, the trachea is extubated in the OR only when the patient is fully awake and has an intact cough/gag reflex, apart from satisfactory extubation criteria. Otherwise, the patients are sedated and shifted to ICU for elective ventilation. The presence of pneumocephalus and brain oedema were found to be the two main reasons for not obeying to verbal commands.
The strategy of prolonged elective ventilation, however, increases the financial cost and subsequently, impact the turnover of major elective surgeries. However, cost of hospitalisation in electively ventilated patients was not estimated in this study as it was not an objective. Beauregard et al. recommended selective, rather than routine use of postoperative ICU care in elective craniotomy patients so as to provide significant reductions in average hospitalisation length and cost. ${ }^{[12]}$ As a protocol, we admit all the postoperative neurosurgical patients to ICU. Subsequently, these patients are shifted to the ward after $12 \mathrm{hrs}$ of uneventful course. Patients requiring elective ventilation are observed for $24 \mathrm{hrs}$ after extubation before being shifted to ward. As evident the ICU and hospital stay gets prolonged with increased duration of postoperative ventilation. Our data suggests that ICU stay of more than $48 \mathrm{hrs}$ was associated with development of pneumonia, subsequent increase in hospital stay and poor neurologic outcome.

There were few limitations to this study. Neurosurgeon's advice, an indication for postoperative MV, is a non-specific terminology. Its use might have resulted in overlapping of certain obvious indications such as intraoperative tight brain, brainstem handling, etc., The surgical skill of the operating neurosurgeon was not taken into consideration which could have affected the surgical outcome.

In conclusion, not every patient operated for intracranial tumour requires postoperative ventilation; hence, effective differentiation is of paramount importance. The decision on timing of tracheal extubation is complex and depends on various systemic and cerebral conditions. Although the neurosurgeon's advice for elective ventilation should not be ignored, but prolonged MV may exacerbate the postoperative morbidities apart from increasing the cost of treatment. Hence, a complete understanding of intraoperative events, cerebral physiology and various factors influencing it during the perioperative period may not be overemphasised.

\section{REFERENCES}

1. Chang DW. Principles of mechanical ventilation. In: Chang DW, editor. Clinical application of mechanical ventilation. $2^{\text {nd }}$ ed. Singapore: Thomson Asia Pvt Ltd. 2005. p. 1-24.

2. Lowes T, Wright J. Anaesthesia and postoperative ventilation. Curr Anaesth Crit Care 2006;17:43-53.

3. Gillespie DJ, Marsh HM, Divertie MB, Meadows JA $3^{\text {rd }}$. Clinical outcome of respiratory failure in patients requiring prolonged (>24 hours) mechanical ventilation. Chest 1986;90:364-9.

4. Bui JQ, Mendis RL, van Gelder JM, Sheridan MM, Wright KM, Jaeger M. Is postoperative intensive care unit admission a prerequisite for elective craniotomy? J Neurosurg 2011;111:1236-41.

5. Coplin WM, Pierson DJ, Cooley KD, Newell DW, Rubenfeld GD. Implications of extubation delay in brain-injured patients 
meeting standard weaning criteria. Am J Respir Crit Care Med 2000;161:1530-6.

6. Basali A, Mascha EJ, Kalfas I, Schubert A. Relation between perioperative hypertension and intracranial hemorrhage after craniotomy. Anesthesiology 2000;93:48-54.

7. Bruder N, Stordeur JM, Ravussin P, Valli M, Dufour H, Bruquerolle B, et al. Metabolic and hemodynamic changes during recovery and tracheal extubation in neurosurgical patients: Immediate versus delayed recovery. Anesth Analg 1999;89:674-8.

8. Schubert A, Mascha EJ, Bloomfield EL, DeBoer GE, Gupta MK, Ebrahim ZY. Effect of cranial surgery and brain tumour size on emergence from anesthesia. Anesthesiology 1996;85:513-21.

9. Dohi S, Okubo N, Kondo Y. Pulmonary oedema after airway obstruction due to bilateral vocal cord paralysis. Can J Anesth 1991;38:492-5.
10. Sharma D, Bithal PK, Dash HH, Chouhan RS, Sookplung P, Vavilala MS. Cerebral autoregulation and $\mathrm{CO} 2$ reactivity before and after elective supratentorial tumour resection. J Neurosurg Anesthesiol 2010;22:132-7.

11. Yasargil MG, Abernathy CD. Microsurgery of CNS tumours IV B. In: Strategies, Tactics and Techniques. Ch. 4. New York: Thieme Medical Publishers; 1996. p. 69-92.

12. Beauregard CL, Friedman WA. Routine use of postoperative ICU care for elective craniotomy: A cost-benefit analysis. Surg Neurol 2003;60:483-9.

How to cite this article: Mahajan C, Rath GP, Sharma MS, Dube SK, Rajagopalan V, Bithal PK. Rate and reasons for elective ventilation in patients undergoing intracranial tumour surgery. J Neuroanaesthesiol Crit Care 2014;1:125-30.

Source of Support: Nil, Conflict of Interest: None declared.

Announcement

\section{Android App}

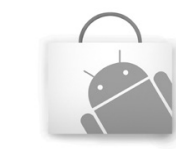

Download Android

A free application to browse and search the journal's content is now available for Android based mobiles and devices. The application provides "Table of Contents" of the latest issues, which are stored on the device for future offline browsing. Internet connection is required to access the back issues and search facility. The application is compatible with all the versions of Android. The application can be downloaded from https:// market.android.com/details?id=comm.app.medknow. For suggestions and comments do write back to us. 